\title{
Antibodies against six human herpesviruses in relation to seven cancers in black South Africans: A case control study
} A Berrington de González*1,2, MI Urban ${ }^{3}$, F Sitass $3,4,5$, N Blackburn ${ }^{6}$, M Hale ${ }^{7}$, M Patel ${ }^{8}$, P Ruff 9 , R Sur ${ }^{10,11}$, R Newton ${ }^{1}$ and V Beral ${ }^{1}$

\begin{abstract}
Address: ${ }^{1}$ Cancer Research UK Epidemiology Unit, University of Oxford, Richard Doll Building, Roosevelt Drive, Headington, Oxford, OX3 7LF,

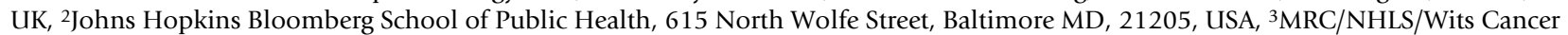
Epidemiology Research Group, National Health Laboratory Service, P.O. Box 1038, Johannesburg 2000, South Africa, ${ }^{4}$ The Cancer Council New South Wales, Sydney, Australia, ${ }^{5}$ The Schools of Public Health, Universities of New South Wales and Sydney, Australia, ${ }^{6}$ National Institute for Communicable Diseases, National Health Laboratory Service, 1 Modderfontein Road, Sandringham 2131, South Africa, ${ }^{7}$ Department of Anatomical Pathology, Chris Hani Baragwanath Laboratory, School of Pathology, University of the Witwatersrand, and National Health Laboratory Service, P.O. Box 1038, Johannesburg 2000, South Africa, ${ }^{8}$ Clinical Haematology Division, Department of Medicine, Chris Hani Baragwanath Hospital and the University of the Witwatersrand, 7 York Road, Parktown, Johannesburg 2193, South Africa, ${ }^{9}$ Division of Medical Oncology, Department of Medicine, Johannesburg Hospital and the University of the Witwatersrand, 7 York Road, Parktown, Johannesburg 2193, South Africa, ${ }^{10}$ Division of Radiation Oncology, Department of Medicine, Johannesburg Hospital and the University of the Witwatersrand, 7 York Road, Parktown, Johannesburg 2193, South Africa and ${ }^{11}$ Hamilton Regional Cancer Centre, 699 Concession Street, Hamilton, ON L8V 5C2, Canada

Email: A Berrington de González* - aberring@jhsph.edu; MI Urban - margaret.urban@nhls.ac.za; F Sitas - freddys@nswcc.org.au; N Blackburn - blackburn_n2004@yahoo.com.au; M Hale - Martin.hale@nhls.ac.za; M Patel - patelmm@medicine.wits.ac.za; P Ruff - pruff@iafrica.com; R Sur - ranjan.sur@hrcc.on.ca; R Newton - Rob.Newton@egu.york.ac.uk; V Beral - PA.Valerie.Beral@cancer.org.uk * Corresponding author
\end{abstract}

Published: 14 September 2006

Infectious Agents and Cancer 2006, I:2 doi:10.1 I86/1750-9378-I-2
Received: 06 June 2006

Accepted: 14 September 2006

This article is available from: http://www.infectagentscancer.com/content/I/I/2

C 2006 de González A et al; licensee BioMed Central Ltd.

This is an Open Access article distributed under the terms of the Creative Commons Attribution License (http://creativecommons.org/licenses/by/2.0), which permits unrestricted use, distribution, and reproduction in any medium, provided the original work is properly cited.

\begin{abstract}
Background: Infections with certain human herpesviruses have been established as risk factors for some cancer types. For example, Epstein-Barr Virus is considered a cause of Burkitt's lymphoma and other immunosuppression related lymphomas, Hodgkin lymphoma, and nasopharyngeal cancer. Several other human herpesviruses have been linked to cancers but the totality of evidence is inconclusive.

Methods: We conducted a systematic sub-study from within an ongoing case control study of adult black South Africans to investigate the relationship between antibodies to six human herpesviruses and seven cancer groups that may be caused by infectious agents. Subjects had incident cancers of the oral cavity $(n=88)$, the cervix $(n=53)$, the prostate $(n=$ 66), Hodgkin lymphoma $(n=83)$, non-Hodgkin lymphoma $(n=80)$, multiple myeloma $(n=94)$ or leukaemia $(n=203)$. For comparison, patients with other cancers $(n=95)$ or cardiovascular disease $(n=101)$ were randomly selected from within the study. Patients were interviewed and their blood was tested for IgG antibodies against HSV-I, HSV-2, VZV, EBVEBNA, CMV and HHV-6 using enzyme linked immunosorbent assays. Because these viruses are highly prevalent in this population, optical density results from the assays were used as an indirect, quantitative measure of antibody level.

Results: There was significant variation in the mean log antibody measures for HSV-2, VZV, CMV and HHV-6 between the disease groups. However, none of the specific cancer groups had significantly higher mean log antibody measures for any of the viruses compared to either control group. In a more detailed examination of seven associations between cancers and herpesviruses for which there had been prior reports, two statistically significant associations were found: a decreasing risk of myeloid leukaemia and an increasing risk of oral cancer with increasing tertiles of antibodies against HHV-6 compared to all other patients ( $p$-trend $=0.03$ and 0.02 , respectively). Odds ratios for the top tertile compared to the bottom tertile were $0.58(95 \% \mathrm{Cl} 0.3-\mathrm{I} .0)$ for myeloid leukaemia and $2.2 \mathrm{I}(95 \% \mathrm{Cl} \mathrm{I} . \mathrm{I}-4.3)$ for oral cancer.
\end{abstract}


Conclusion: In this population, using these tests for lgG, neither mean antibody measure nor high antibody measure against human herpesviruses I-6 was strongly associated with any of the seven cancer groups. However, we may not have had sufficient power to detect weak associations or associations with a sub-type of cancer if they were present.

\section{Background}

Infection with certain types of human herpesviruses has been established as a cause of several cancers. These include Epstein-Barr Virus (EBV) for Burkitt's lymphoma and other immunosuppression related lymphomas, Hodgkin lymphoma, and nasopharyngeal cancer [1]; and human herpesvirus 8 (HHV-8) for Kaposi's sarcoma [2]. These cancers are rare responses to the presence of these widespread viruses.

Several human herpesviruses have been linked to other cancers although the totality of evidence is inconclusive. For example herpes simplex virus type 1 (HSV-1) has been associated with oral cancer[3] and herpes simplex virus type 2 (HSV-2) with cervical cancer in women who are coinfected with specific human papillomavirus types[4]. Human herpesvirus type 6 (HHV-6) has been linked to Hodgkin lymphoma [5], acute myeloid leukaemia [6] and oral cancer [7]. In addition it has been suggested that prostate cancer [8] and multiple myeloma [9] may have infectious causes.

Our group previously found that high antibody levels to HHV8 are highly correlated to the diagnosis of Kaposi's sarcoma [2]. We therefore designed a study to examine, in a systematic way, antibody levels to six of the herpesviruses (HSV-1, HSV-2, Varicella Zoster (VZV), EBV, cytomegalovirus (CMV) and HHV-6) in relation to seven cancer groups for which there is some evidence of an infectious cause (oral, cervical, prostate, Hodgkin lymphoma, non-Hodgkin lymphoma, multiple myeloma and leukaemia). The study was part of a larger case-control study of the causes of cancer in black South Africans, which was conducted in public hospitals that treat cancer in greater Johannesburg, South Africa $[2,10]$. Since most human herpesviruses are highly prevalent, and PCR on biopsy samples is unrealistic in this setting, we used quantitative measures of anti-human herpesvirus antibodies from enzyme linked immunosorbent assays (ELISAs). In addition we examined the relationships between demographic and lifestyle factors and antibody levels against these viruses, as little is known about these viruses in this population.

\section{Methods}

\section{Study Participants}

The study population has been described previously [2,10]. Briefly, between March 1995 and February 1999 trained nurses interviewed adult black patients with newly diagnosed cancer at tertiary government hospitals in Johannesburg (Chris Hani-Baragwanath, Hillbrow, and Johannesburg General Hospitals). A standard questionnaire, administered in the language of the patient (usually an Nguni or Sotho group language), was used. Questions were asked about socio-demographic factors and behavioural characteristics including age, sex, birthplace, residence, level of education, tobacco and alcohol use, and reproductive and lifetime sexual history.

Blood was collected from $84 \%$ of patients at the time of interview and prior to commencing treatment. The remainder were too ill, had collapsed veins, or refused consent. All interviewed patients with oral cancer $(\mathrm{n}=$ 88), Hodgkin lymphoma ( $\mathrm{n}=83)$, non-Hodgkin lymphoma $(n=80)$, multiple myeloma $(n=94)$ and leukaemia $(n=203)$ and a random sample of subjects with cervical $(n=53)$ and prostate cancer $(n=66)$ were included in the study if they had provided a blood sample. Controls were from two groups of patients attending the same hospitals: a group of patients with other cancers $(\mathrm{n}=95)$ and a group with cardiovascular diseases $(\mathrm{n}=$ 101). The controls were selected randomly within sex and age bands, and frequency matched to the cancer cases as a whole according to five-year age-groups and sex. Diagnoses of cancer were established, where appropriate, by histology, haematology, or cytology. The study was approved by the Committee for Research on Human Subjects (Medical) of the University of the Witwatersrand, and informed consent was obtained from all patients.

\section{Laboratory methods}

After coagulation, specimens were centrifuged to obtain serum. The serum specimens were aliquotted and stored at $-20^{\circ}$ to $-30^{\circ} \mathrm{C}$. HIV testing was carried out by the Serology Laboratory of the South African Institute for Medical Research (now the National Health Laboratory Service), using commercial ELISA kits. Early testing was for HIV-1 only; later testing for both HIV-1 and HIV-2. Patients with borderline results were considered to be HIV negative.

Herpes virus antibody testing was done at the South African National Institute for Virology (now the National Institute for Communicable Diseases) from January to April 2000. Throughout the testing period specimens were kept at $4^{\circ} \mathrm{C}$. The commercially available kits used were: Eurogenetics for HSV-1, HSV-2 and CMV; Clark Laboratories for VZV and EBV nuclear antigen-1(EBNA); and PanBio for HHV-6. These were selected following 
apreliminary study which demonstrated good quantitative performance with South African sera. The test for EBNA IgG used "recombinant EBNA-1 antigen". All other kits used unspecified antigens.

Patients' sera were randomly allocated to the 96-well microplates to minimise potential bias due to systematic differences between the plates or day on which the plates were run. Each specimen was run in a single well. There were a total of 12 plates for each assay and in general two plates were run each day. Repeatability of each assay was investigated through replicate measures on a pool of sera samples with high antibody measures against each virus. Test results were initially in optical density units which are proportional to the concentration of antibody i.e. the antibody titre. The CMV kit had a calibration curve giving final results in antibody units $/ \mathrm{ml}$. The remaining kits contained standards used to determine a cut-off for positivity. Results are reported as ratios to the manufacturer's cut-off value. Throughout this paper we use the general term "antibody measure" to refer to the manufacturer's result, regardless of whether it is in $\mathrm{AU} / \mathrm{ml}$ or "times cut-off".

\section{Statistical Analysis for the control samples}

There were several potential sources of variability in each assay such as antigen binding within or between plates, washing conditions between binding steps and laboratory conditions such as the temperature, humidity, and light. For each of the herpesviruses the logarithm (log) antibody measure was computed for the control pool and analysis of variance was used to estimate the magnitude of the components of variance for each of the sources of variability.

\section{Statistical Analysis of the Patient Data}

To examine the relation between antibodies against the human herpesviruses 1-6 and cancer the mean log antibody measures for each of the nine disease groups were compared using analysis of variance. Least square mean $\log$ antibody measures were calculated for each disease group with adjustment for age, sex, HIV status, and the day and the plate on which the assay was run. Paired comparisons were conducted between each cancer group and each of the two control groups (other cancers and cardiovascular disease patients) with Bonferroni adjustments for multiple comparisons. Where positive associations had previously been found (see Introduction [1-7]), the specific hypotheses were investigated in more detail by comparing the specified cancer group to all the other patients combined with respect to tertiles of log antibody measure. Odds ratios were calculated using logistic regression with adjustment for age, sex, HIV status and the day and the plate on which the assay was run.
Since little is known about risk factors for infection with human herpesviruses in this population, we examined the relationship between antibody measure and a number of socio-demographic variables using analysis of variance with adjustment for each of these variables as well as cancer group, day of assay, and assay plate. Due to the number of comparisons that were made these analyses were examined at the 0.01 significance level.

All analyses were carried out using SAS statistical software [11].

\section{Results}

In total 667 patients with the seven specific cancers of interest were included in the study. A further 95 patients with other types of cancer and 101 patients with cardiovascular diseases were included as control subjects. Demographic characteristics of the study members are shown in Table 1.

The results from the analysis of the control samples suggested that for all assays (except for HSV-1 and HSV-2) the most important source of systematic variation was the day on which the plates were assayed, whereas for the HSV-1 and HSV-2 assays it was the plates themselves (data not shown). As the plate and day on which each patient's sample was tested had been recorded, variation between days and between plates was controlled for in the main analyses by adjusting for these factors.

Crude adult prevalence rates in the study, determined using the manufacturer's criteria for positivity, were: $99 \%$ for HSV $-1 ; 59 \%$ for HSV-2 (52\% in males and 68\% in females); $97 \%$ for VZV; $96 \%$ for EBV (EBNA antigen); $99 \%$ for $\mathrm{CMV}$; and $90 \%$ for HHV-6 (88\% in males and $93 \%$ in females).

\section{Associations with cancer types}

There was statistically significant variation between the nine disease groups in mean log antibody measure for HSV-2 ( $<<0.0001)$, VZV $(p<0.0001), \operatorname{CMV}(\mathrm{p}=0.009)$ and HHV-6 ( $\mathrm{p}<0.0001)$ (Figure 1). Patients with multiple myeloma had lower mean log antibody measures for almost all these herpesviruses compared to other patients. When patients with multiple myeloma were excluded, significant heterogeneity in mean log antibody measures between the eight remaining disease groups was still present for HSV-2 $(\mathrm{p}=0.0007)$ and HHV-6 $(\mathrm{p}=0.004)$.

In paired comparisons (with Bonferroni adjustments), patients with multiple myeloma had significantly lower mean $\log$ IgG antibody measures for the response to VZV, CMV and HHV- 6 compared to patients in the 'other cancer' group ( $\mathrm{p}<0.0001,0.02$ and $\mathrm{p}<0.0001$ respectively) and for HSV-2, VZV and HHV-6 compared to patients 


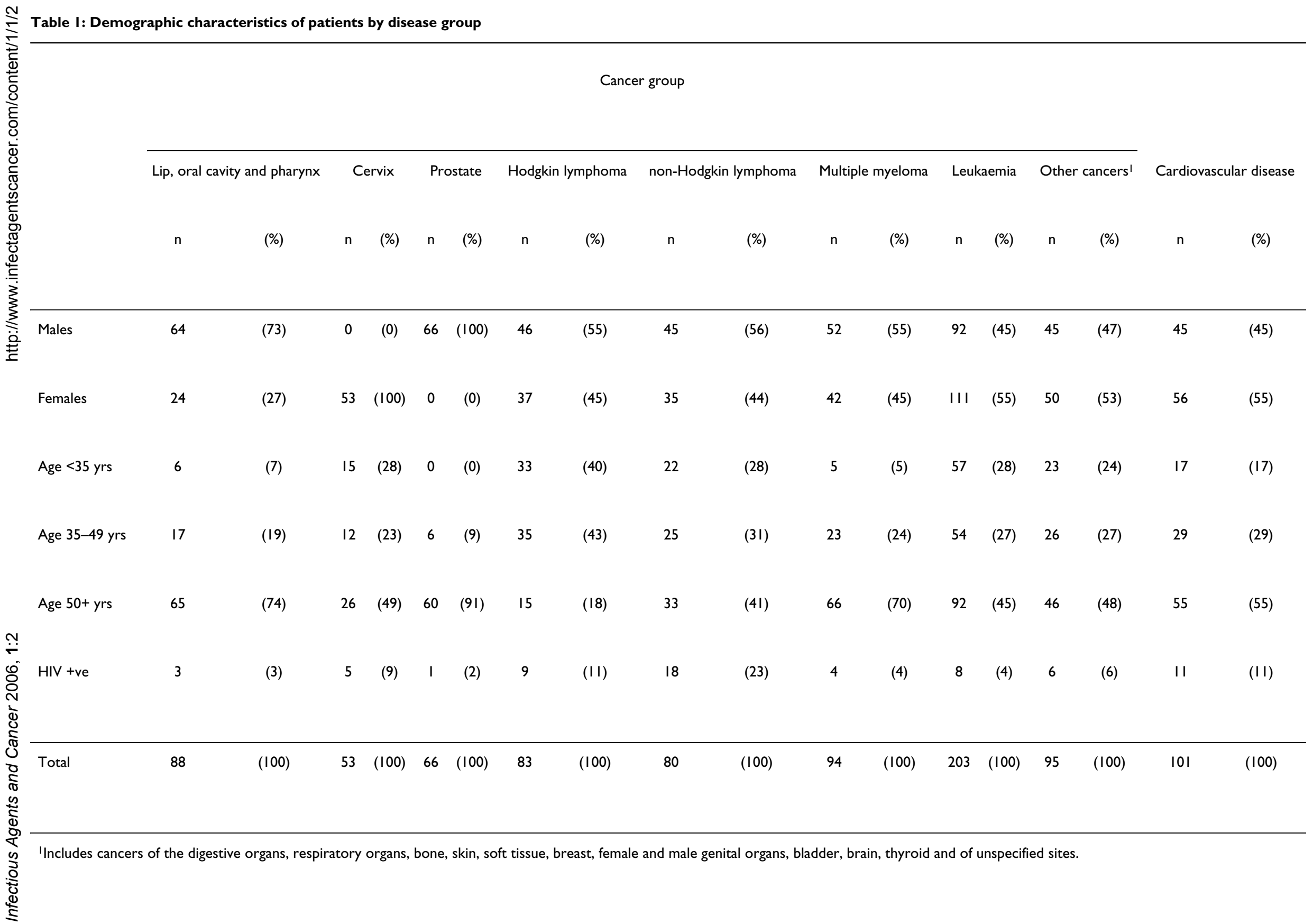




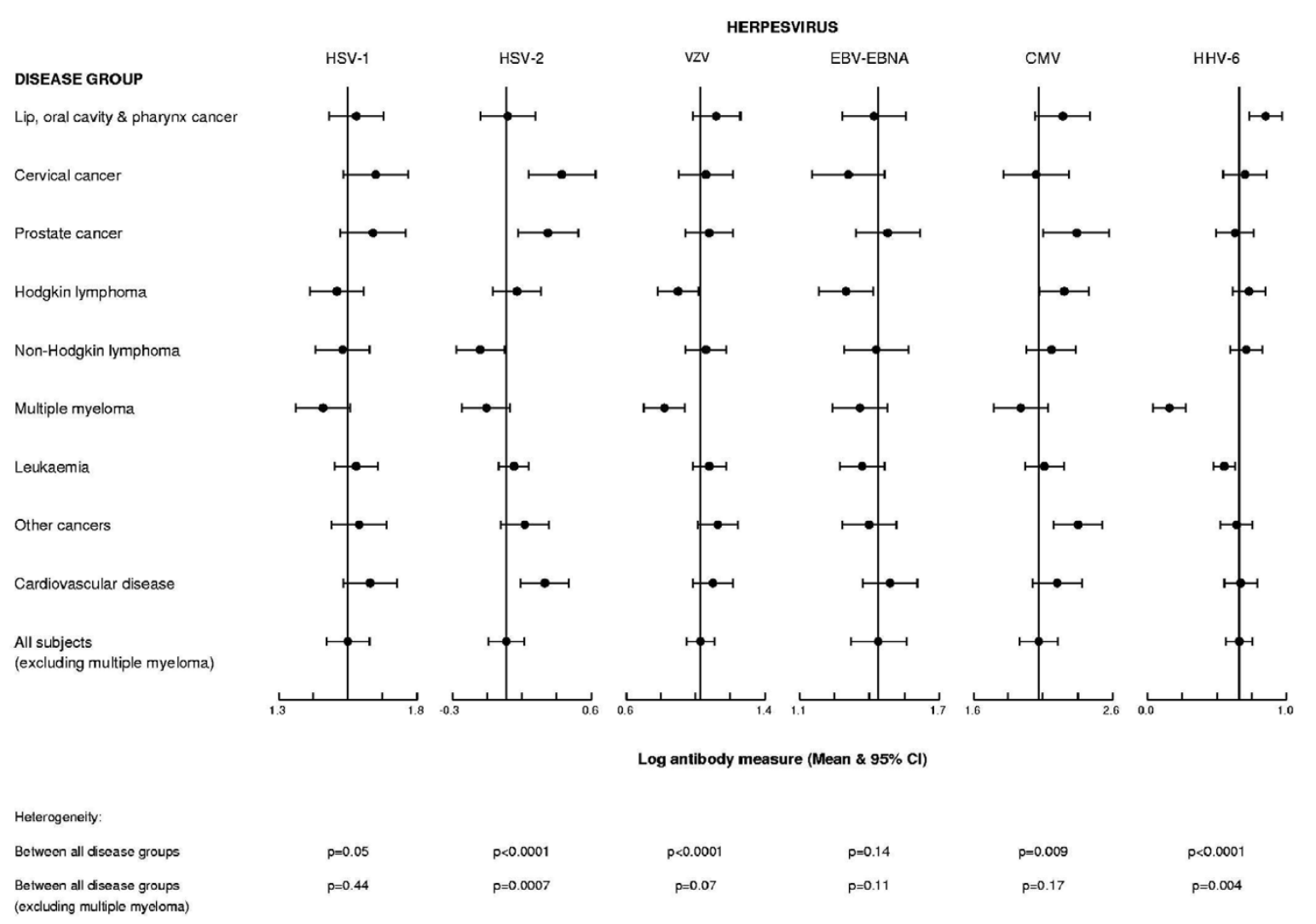

Figure I

Distribution of mean log antibody measures (and $95 \% \mathrm{Cl}$ ) by disease group for herpesviruses $\mathrm{I}-6$.

with cardiovascular diseases $(\mathrm{p}=0.01,0.0003$ and $<0.0001$ respectively). Because of their systematically lower antibody measures, multiple myeloma patients were excluded from further analyses. Patients with nonHodgkin lymphoma had significantly lower mean log antibody measures in response to HSV-2 compared to the non-cancer patients $(\mathrm{p}=0.004)$. None of the specific cancer groups had significantly higher mean log antibody measures for any of the viruses compared to either control group.

To further investigate the positive associations reported in previous studies (EBV and Hodgkin lymphoma; EBV and non-Hodgkin lymphoma; HSV-1 and oral cancer; HSV-2 and cervical cancer; HHV-6 with oral cancer, Hodgkin lymphoma and acute myeloid leukaemia) the log antibody measures were divided into tertiles according to the levels in allpatientsexcluding those with multiple myeloma. The control group was defined in this way for these analyses because in general mean antibody levels did not vary significantly across disease groups after exclusion of subjects with multiple myeloma (Figure 1), and including more patients increased the power of these analyses. Only oral cancer showed a statistically significant trend of risk with increasing HHV-6 tertiles $(\mathrm{p}=0.02)$, odds ratio for the top tertile compared to all other subjects $=2.21(95 \%$ CI 1.1 - 4.3) (Table 2). All the oral cancer patients were positive for HHV-6 antibodies by the manufacturer's cutoff. Only two subjects had undifferentiated nasopharyngeal cancer so it was not possible to investigate the relationship between this sub-type of oral cancers and EBV. Neither was it possible to separate acute myeloid leukaemias from chronic myeloid leukaemias with the information that was available, but there was some evidence of a decreasing trend in risk of myeloid leukaemia with increasing tertiles of HHV-6 ( $\mathrm{p}=0.03)$; odds ratio for the top tertile compared to all other subjects $=0.58(95 \% \mathrm{CI}$ $0.3-1.0)$. 
Table 2: Odds ratios (OR) and $95 \%$ confidence intervals $(\mathrm{Cl})$ for specific cancers ${ }^{1}$ versus all other subjects (controls) ${ }^{2}$ by tertile of virus antibody measure in the controls

\begin{tabular}{|c|c|c|c|c|c|}
\hline Virus and cancer group & Control tertiles & Cases/Controls & OR & $95 \% \mathrm{Cl}$ & P-trend \\
\hline \multirow[t]{3}{*}{ HSV-I and Oral cancer } & $0-5.04$ & $23 / 200$ & 1.00 & & \\
\hline & $5.05-6.07$ & $26 / 199$ & 1.06 & $(0.6-2.0)$ & \\
\hline & $6.08+$ & $31 / 199$ & 1.28 & $(0.7-2.3)$ & 0.40 \\
\hline \multirow[t]{3}{*}{ HSV-2 and Cervical cancer } & $0-1.08$ & ||$/ 9 \mid$ & 1.00 & & \\
\hline & $1.09-2.17$ & $1 \mid / 91$ & 0.90 & $(0.4-2.2)$ & \\
\hline & $2.18+$ & $23 / 90$ & 1.82 & $(0.8-4.1)$ & 0.10 \\
\hline \multirow[t]{3}{*}{ EBV-EBNA and non-Hodgkin lymphoma } & $0-3.81$ & $24 / 208$ & 1.00 & & \\
\hline & $3.82-5.29$ & $31 / 203$ & 1.32 & $(0.7-2.4)$ & \\
\hline & $5.30+$ & $22 / 211$ & 0.87 & $(0.4-1.8)$ & 0.80 \\
\hline \multirow[t]{3}{*}{ EBV-EBNA and Hodgkin lymphoma } & $0-3.87$ & $33 / 211$ & 1.00 & & \\
\hline & $3.88-5.31$ & $18 / 208$ & 0.49 & $(0.3-0.96)$ & \\
\hline & $5.32+$ & $21 / 208$ & 0.66 & $(0.3-1.4)$ & 0.19 \\
\hline \multirow[t]{3}{*}{ HHV-6 and Oral cancer } & $0-1.59$ & $16 / 195$ & 1.00 & & \\
\hline & $1.60-2.43$ & $28 / 194$ & 1.83 & $(0.9-3.6)$ & \\
\hline & $2.44+$ & $31 / 195$ & 2.21 & $(1.1-4.3)$ & 0.02 \\
\hline HHV-6 and Hodgkin lymphoma & $0-1.58$ & $21 / 172$ & 1.00 & & \\
\hline \multirow[t]{2}{*}{ (excluding oral cancers) } & $1.59-2.40$ & $17 / 174$ & 0.87 & $(0.4-1.7)$ & \\
\hline & $2.41+$ & $28 / 172$ & 1.32 & $(0.7-2.5)$ & 0.39 \\
\hline HHV-6 and Myeloid Leukaemia & $0-1.61$ & $47 / \mid 31$ & 1.00 & & \\
\hline \multirow[t]{2}{*}{ (excluding oral cancers) } & $1.62-2.59$ & $52 / 134$ & 1.16 & $(0.7-1.9)$ & \\
\hline & $2.60+$ & $26 / 131$ & 0.58 & $(0.3-1.0)$ & 0.03 \\
\hline
\end{tabular}

IFor associations that had been reported previously.

2 The control group in these analyses included all other subjects, except patients with multiple myeloma, because in general mean antibody levels did not vary significantly across disease groups after exclusion of subjects with multiple myeloma (Figure I), and including all patients increased the power of these analyses.

All analyses are adjusted for age group, sex, day of assay and assay plate.

Association with age, sex and socio-demographic factors Possible determinants of high antibody measures to these herpesviruses, such as age and sex and socio-demographic factors, were investigated in an analysis of variance using data from all the patients except those with multiple myeloma (Table 3). No risk factors were identified as significantly influencing high log antibody measures in response to HSV-1. For HSV-2 mean log antibody measures increased with age ( $\mathrm{p}$-trend $=0.003$ ), were higher in women $(p<0.0001)$, increased with number of sexual partners ( $\mathrm{p}$-trend $=0.01$ ) and were lower in single people (p-heterogeneity $=0.005)$. No statistically significant associations were found for VZV, or EBV-EBNA. Mean log antibody measure for response to CMV increased significantly with age ( $\mathrm{p}$-trend $=0.006$ ) and was also higher in females than in males $(p<0.0001)$. Similarly, the mean log antibody measure for HHV-6 antibodies was significantly higher in females than males $(\mathrm{p}=0.0002)$.

\section{Discussion}

Herpesvirus infections are highly prevalent in human populations. They usually produce transient illness or inapparent infection and remain latent in the host. In general, re-activation of the latent infection, re-infection, or viral persistence will cause the established IgG antibody levels to rise. In Burkitt's lymphoma it has been shown that raised levels of IgG were present months before clin- ical onset [12]. Antibodies to HHV-8 are also found many months prior to the diagnosis or Kaposi's sarcoma [13] and the diagnosis is highly correlated to high HHV-8 antibody levels [2]. It is therefore reasonable to speculate that raised antibody levels to other viruses may be associated with the development of other cancers.

We conducted a systematic study of the relationship between antibodies against human herpesviruses 1-6 and seven cancer groups in adult black South Africans. Our results suggest that, in this population, neither mean ELISA IgG antibody measure to the antigens used nor high antibody measures against these six human herpesviruses were strongly associated with any of the seven cancer groups. Although we do not think that the variability in the quantitative assay results concealed any strong associations, we may not have had sufficient power to detect weak associations (for example, odds ratios <2.0) or associations with a sub-type of cancer if they were present.

Though there was no evidence of a significant association between high EBV-EBNA antibody measure and nonHodgkin lymphoma or Hodgkin lymphoma, EBV may be a causative factor of certain types of lymphomas only, including Burkitt's lymphoma and some immunosupression associated lymphomas [1]. As mentioned above, the current study was not powered to detect associations in 
Table 3: Mean log antibody measure (and standard error) for human herpesviruses I-6 according to age at diagnosis, sex and other socio-demographic factors.

\begin{tabular}{|c|c|c|c|c|c|c|c|}
\hline \multirow[t]{2}{*}{ Risk factor } & & \multicolumn{6}{|c|}{ Virus } \\
\hline & & HSV-I & HSV-2 & VZV & EBV-EBNA & CMV & HHV-6 \\
\hline \multirow[t]{4}{*}{ Age } & $<20$ & $1.55(0.11)$ & $-0.19(0.20)$ & $1.06(0.12)$ & $1.52(0.15)$ & $1.79(0.21)$ & $0.64(0.15)$ \\
\hline & $20-39$ & $1.61(0.04)$ & $0.25(0.08)$ & $1.06(0.05)$ & $\mathrm{I} .44(0.07)$ & $2.19(0.12)$ & $0.70(0.06)$ \\
\hline & $40-59$ & $1.61(0.04)$ & $0.36(0.07)$ & $1.07(0.05)$ & $1.40(0.07)$ & $2.26(0.07)$ & $0.74(0.06)$ \\
\hline & $60+$ & $1.60(0.05)$ & $0.48(0.08)$ & I.II (0.06) & $\mathrm{I} .47(0.07)$ & $2.41(0.09)$ & $0.66(0.05)$ \\
\hline p-value & & 0.92 & 0.003 & 0.41 & 0.77 & 0.006 & 0.52 \\
\hline \multirow[t]{2}{*}{ Sex } & Males & $1.59(0.05)$ & $0.05(0.08)$ & $1.03(0.06)$ & I.43 (0.07) & $1.98(0.09)$ & $0.59(0.06)$ \\
\hline & Females & $1.60(0.05)$ & $0.40(0.08)$ & I.II (0.06) & $1.48(0.07)$ & $2.34(0.10)$ & $0.78(0.06)$ \\
\hline p-value & & 0.91 & $<0.0001$ & 0.09 & 0.44 & $<0.0001$ & 0.0002 \\
\hline \multirow[t]{2}{*}{ Smoking } & Never & $1.60(0.04)$ & $0.21(0.08)$ & $1.03(0.05)$ & $\mathrm{I} .45(0.07)$ & $2.16(0.09)$ & $0.67(0.06)$ \\
\hline & Ever & $1.59(0.05)$ & $0.24(0.09)$ & $1.11(0.06)$ & $1.46(0.08)$ & $2.17(0.10)$ & $0.70(0.06)$ \\
\hline p-value & & 0.91 & 0.56 & 0.07 & 0.96 & 0.94 & 0.34 \\
\hline \multirow[t]{3}{*}{ Education } & $<$ Grade 3 & $1.59(0.05)$ & $0.30(0.09)$ & $1.10(0.06)$ & I.4I (0.08) & $2.18(0.10)$ & $0.74(0.07)$ \\
\hline & Grade 3-7 & $1.64(0.05)$ & $0.24(0.08)$ & $1.09(0.05)$ & $1.47(0.07)$ & $2.13(0.09)$ & $0.69(0.06)$ \\
\hline & Grade 8+ & $1.55(0.05)$ & $0.14(0.09)$ & $1.04(0.06)$ & $1.49(0.08)$ & $2.18(0.10)$ & $0.64(0.07)$ \\
\hline p-value & & 0.53 & 0.09 & 0.26 & 0.18 & 0.99 & 0.14 \\
\hline \multirow[t]{2}{*}{ Area of birth } & Urban & $1.61(0.05)$ & $0.28(0.09)$ & $1.10(0.06)$ & $\mathrm{I} .46(0.07)$ & $2.14(0.10)$ & $0.70(0.06)$ \\
\hline & Rural & $1.57(0.04)$ & $0.18(0.08)$ & $1.05(0.05)$ & $1.45(0.07)$ & $2.19(0.09)$ & $0.67(0.07)$ \\
\hline p-value & & 0.29 & 0.13 & 0.16 & 0.67 & 0.48 & 0.45 \\
\hline \multirow[t]{2}{*}{ Area living } & Urban & I.59 (0.04) & $0.19(0.07)$ & $1.08(0.05)$ & $\mathrm{I} .45(0.07)$ & $2.17(0.09)$ & $0.71(0.06)$ \\
\hline & Rural & $1.59(0.05)$ & $0.27(0.09)$ & $1.07(0.06)$ & $1.46(0.08)$ & $2.15(0.11)$ & $0.67(0.06)$ \\
\hline p-value & & 0.99 & 0.35 & 0.83 & 0.76 & 0.85 & 0.53 \\
\hline \multirow[t]{2}{*}{ HIV } & +ve & I.53(0.06) & $0.27(0.11)$ & $1.12(0.07)$ & I.43(0.09) & $2.22(0.12)$ & $0.7 \mid(0.08)$ \\
\hline & -ve & $1.66(0.04)$ & $0.18(0.07)$ & $1.02(0.05)$ & $1.48(0.06)$ & $2.11(0.08)$ & $0.67(0.05)$ \\
\hline p-value & & 0.03 & 0.35 & 0.10 & 0.52 & 0.34 & 0.66 \\
\hline No. of & $0-2$ & $1.60(0.05)$ & $0.12(0.08)$ & $1.02(0.06)$ & I.47 (0.07) & $2.12(0.09)$ & $0.64(0.06)$ \\
\hline sexual & $3-5$ & $1.58(0.05)$ & $0.25(0.08)$ & $1.05(0.06)$ & I.4I (0.07) & $2.18(0.09)$ & $0.75(0.06)$ \\
\hline partners & $6+$ & $1.60(0.05)$ & $0.31(0.09)$ & $1.15(0.06)$ & I.49 (0.08) & $2.19(0.11)$ & $0.67(0.07)$ \\
\hline p-value & & 0.96 & 0.01 & 0.03 & 0.96 & 0.34 & 0.26 \\
\hline Marital & Single & I.58 (0.04) & $0.06(0.08)$ & $1.04(0.05)$ & $\mathrm{I} .45(0.07)$ & $2.03(0.09)$ & $0.65(0.06)$ \\
\hline \multirow[t]{3}{*}{ status } & Married & $1.62(0.05)$ & $0.22(0.08)$ & I.II (0.05) & $\mathrm{I} .43(0.07)$ & $2.16(0.09)$ & $0.67(0.06)$ \\
\hline & Widowed & $1.59(0.06)$ & $0.20(0.11)$ & $\mathrm{I} .04(0.07)$ & I.4I (0.09) & $2.17(0.12)$ & $0.66(0.08)$ \\
\hline & Separated & $1.58(0.07)$ & $0.42(0.12)$ & $1.10(0.08)$ & $1.54(0.10)$ & $2.29(0.14)$ & $0.77(0.09)$ \\
\hline p-value & & 0.64 & 0.005 & 0.45 & 0.52 & 0.26 & 0.34 \\
\hline
\end{tabular}

Multivariate analysis of variance excluding patients with multiple myeloma and adjusted for all factors above plus disease group, plate and day of assay. P-values are for trend tests where appropriate and otherwise for heterogeneity.

small sub-groups. However, our results suggest that high IgG antibody measures against EBV-EBNA do not appear to be relevant in most lymphomas in this population. Several other associations between herpesviruses and cancers, which have been reported less consistently in the literature, were not clearly evident in the current study including: HSV-1 and oral cancer; HSV-2 and cervical cancer; HHV-6 and Hodgkin lymphoma; and HHV-6 and myeloid leukaemia.

There was evidence of an increased risk of oral cancer for subjects in the highest compared to lowest tertile of antibodies against HHV-6 compared to other subjects (OR = $2.21,95 \%$ CI $1.1-4.3)$, and a trend of increasing risk across the tertiles $(\mathrm{p}=0.02)$. A similar study in India of patients with Hodgkin lymphoma, non-Hodgkin lymphoma, oral cancer and healthy controls found that patients with oral cancers had elevated levels of HHV-6 antibodies compared to the healthy controls [7]. As far as we are aware no additional studies have published results on the association between HHV-6 and oral cancer, but since two studies have found a positive relationship further research is warranted. There was also an unexpected small decrease in the risk of myeloid leukaemia in patients with the highest tertile of antibodies against HHV-6 (OR = 0.58, 95\% CI $0.3-1.0)$. An increased risk of acute myeloid leukaemia and HHV-6 has been reported previously in some studies $[6,14,15]$, although other studies have not found evidence of such an association $[16,17]$.

The retrospective design on this study means that it is possible that the cancer affected the patient's antibody levels and therefore that the antibody levels reflect a conse- 
quence rather than a cause of the cancer. There was evidence of this with respect to the multiple myeloma patients, who had lower than average mean log antibody measures for response to all six herpesviruses. This may be because myeloma is an over-production of a single immunoglobulin class, which could in turn down-regulate production of other immunoglobulins/antibodies. None of the other disease groups had a systematically higher or lower log antibody measures for response to all six of the herpesviruses. However, it remains a possibility that the positive association between raised HHV-6 antibody levels and the risk of oral cancer could have been caused by opportunistic reactivations due to cancer-associated immunosuppression. Future studies should preferably be conducted with prospectively collected blood samples.

Little is known about the determinants of infection with and antibody response to herpesviruses 1-6 in the population studied. In an analysis of associations with age, sex and lifestyle factors there was evidence that, as expected, HSV-2 antibody measures increased with increasing number of sexual partners. In addition, antibody measures to both HSV-2 and CMV were significantly higher in older age groups, presumably reflecting an increasing number of re-infections, and in women. For HHV-6, which is probably transmitted via saliva [18], antibody measures were also found to be higher in women than in men. Several other studies have reported similar findings for HHV-6 $[19,20]$ although in the study in West Africa and the Caribbean, this was only true for children [19]. Primary HHV-6 acquisition has been found to be associated with female sex [21]. HIV positive patients in our study did not have significantly higher average antibody measures in response to any of these herpesviruses.

As quantitative methods have not previously been widely used in epidemiological studies of herpesviruses we included a quality control analysis to assess the repeatability of the assay results. We found systematic variability that could be attributed to differing laboratory conditions and for HSV-1 and HSV-2 variability attributable to either the binding of the assay components to the plates or calibration control samples that were not effective at reducing the variation between plates. For quantitative assessments improvements to the kit techniques which we used are needed, possibly using different antigens. In the current study we randomised the patients' samples to the plates so that the systematic differences will not have biased our results and took account of these systematic sources of variability in the analyses by adjusting for the plate and day on which the assay was run.

\section{Conclusion}

In the black adult population of greater Johannesburg neither mean IgG antibody measure nor high antibody meas- ures against human herpesvirsues $1-6$ were strongly associated with any of the seven selected cancer groups. We do not think that the variability in the quantitative assay results concealed any strong associations, but we may not have had sufficient power to detect weak associations or associations with a sub-type of cancer. The finding of a small increased risk for oral cancer with increased antibodies to HHV-6 merits further exploration.

\section{Authors' contributions}

$\mathrm{VB}$ and FS conceived of and designed the study. MU was involved in the design of the plate template for serological testing, in co-ordination of the serological testing, and in verification of patient data. NB participated in the study design, selection of test kits and design of the plate template for serological testing as well as supervision of the serological testing. MH provided pathology reports. MP, $\mathrm{PR}$, and RS supplied patients for this study. $\mathrm{AB}$ conducted the statistical analysis and drafted the manuscript. $\mathrm{VB}$, $\mathrm{MU}, \mathrm{RN}$ and FS were involved in the statistical analysis and critical revision of the manuscript.

\section{Acknowledgements}

During the study period the Cancer Epidemiology Research Group was supported by the South African Medical Research Council, the National Health Laboratory Service (formerly South African Institute for Medical Research), the University of the Witwatersrand, the Cancer Association of South Africa and Cancer Research UK.

We are indebted to: Sr. Gloria Mokwatle and colleagues for carrying out the interviews; Ms. F. Mngomezulu and Ms. H. Mathabatha for data coding and entry; Mrs. Lettie Bester for specimen preparation; Ms. Diana Bull for case selection and specimen randomisation; Ms. Lorraine Cranston and Ms. Jane Franz at the South African National Institute for Communicable Diseases (formerly National Institute for Virology) for carrying out the laboratory tests; the clinicians and nursing staff at Chris Hani Baragwanath, Hillbrow, and Johannesburg Hospitals for granting access to clinics, wards, and records; and the patients for their participation in this study.

\section{References}

I. International Agency for Research on Cancer: Epstein-Barr virus and Kaposi's sarcoma herpesvirus/human herpesvirus 8 Lyon: World Health Organization; 1997.

2. Sitas F, Carrara H, Beral V, Newton R, Reeves G, Bull D, Jentsch U, Pacella-Norman R, Bourboulia D, Whitby D, Boshoff C, Weiss R: Antibodies against human herpesvirus 8 in black South African patients with cancer. NEJM 1999, 340:1863-I87I.

3. Schildt EB, Eriksson M, Hardell L, Magnuson A: Oral infections and dental factors in relation to oral cancer: a Swedish case-control study. Eur J Cancer Prev 1998, 7:201-206.

4. Smith JS, Herrero R, Bosetti C, Munoz N, Bosch FX, Eluf-Neto J, Castellsague X, Meijer CJ, Van Den Brule AJ, Franceschi S, Ashley R: Herpes simplex virus-2 as a human papillomavirus cofactor in the etiology of invasive cervical cancer. J Natl Cancer Inst 2002, 94:1604-1613.

5. Alexander FE, Daniel CP, Armstrong AA, Clark DA, Onions DE, Cartwright RA, Jarrett RF: Case clustering, Epstein-Barr virus Reed-Sternberg cell status and herpes virus serology in Hodgkin's disease: results of a case-control study. Eur J Cancer | 995, 3 | A: | 479-|486.

6. Gentile G, Mele A, Ragona G, Faggioni A, Zompetta C, Tosti ME, Visani G, Castelli G, Pulsoni A, Monarca B, Martino P, Mandelli F: Human herpes virus- 6 seroprevalence and leukaemias: a 
case-control study. GIMEMA (Gruppo Italiano Malattie Ematologiche dell' Adulto). Br J Cancer 1999, 80:II03-I I06.

7. Shanavas KR, Kala V, Vasudevan DM, Vijayakumar T, Yadav M: AntiHHV-6 antibodies in normal population and in cancer patients in India. J Exp Pathol 1992, 6:95-105.

8. Strickler HD, Goedert J]: Sexual behavior and evidence for an infectious cause of prostate cancer. Epidemiol Rev 200I, 23:|44-I5I.

9. Gao SJ, Alsina M, Deng JH, Harrison CR, Montalvo EA, Leach CT, Roodman GD, Jenson HB: Antibodies to Kaposi's sarcoma-associated herpesvirus (human herpesvirus 8 ) in patients with multiple myeloma. J Infect Dis 1998, I 78:846-849.

10. Sitas F, Pacella-Norman R, Carrara H, Patel M, Ruff $P$, Sur R, Jentsch $U$, Hale M, Rowji P, Saffer D, Connor M, Bull D, Newton R, Beral V: The spectrum of HIV-I related cancers in South Africa. Int J Cancer 2000, 88:489-492.

II. SAS Institute Inc: SAS. Cary, NC, USA 1999, version 8.2:

12. De-Thé G, Geser A, Day NE, Tukei PM, Williams EH, Beri DP, Smith PG, Dean AG, Bornkamm GW, Feorino P, Henle W: Epidemiological evidence for causal relationship between Epstein-Barr virus and Burkitt's lymphoma from Ugandan prospective study. Nature 1978, 274:756-761.

13. Gao S-J, Kingsley L, Hoover DR, Spira TJ, Rinaldo CR, Saah A, Phair J Detels R, Parry P, Chang Y, Moore PS: Sero conversion to antibodies against Kaposi's sarcoma-associated herpesvirusrelated latent nuclear antigens before the development of Kaposi's sarcoma. NEJM 1996, 335:233-24I.

14. Clark DA, Alexander FE, McKinney PA, Roberts BE, O'Brien C, Jarrett RF, Cartwright RA, Onions DE: The seroepidemiology of human herpesvirus-6 (HHV-6) from a case-control study of leukaemia and lymphoma. Int / Cancer 1990, 45:829-833.

15. Salonen MJ, Siimes MA, Salonen EM, Vaheri A, Koskiniemi M: Antibody status to HHV-6 in children with leukaemia. Leukemia 2002, 16:716-7|9.

16. Levine PH, Ablashi DV, Saxinger WC, Connelly RR: Antibodies to human herpes virus-6 in patients with acute lymphocytic leukemia. Leukemia 1992, 6:|229-|231.

17. Schlehofer B, Blettner M, Geletneky K, Haaf HG, Kaatsch P, Michaelis J, Mueller-Lantzsch N, Niehoff D, Winkelspecht B, Wahrendorf J, Schlehofer JR: Sero-epidemiological analysis of the risk of virus infections for childhood leukaemia. Int J Cancer 1996, 65:584-590.

18. Mukai T, Yamamoto T, Kondo T, Kondo K, Okuno T, Kosuge H, Yamanishi K: Molecular epidemiological studies of human herpesvirus 6 in families. J Med Virol 1994, 42:224-227.

19. Cleghorn FR, Maybank KA, Jack N, Pate E, Mingle J, Levine PH, Manns A: Comparison of HHV-6 antibody titers in West Africa and the Caribbean. Ann Epidemiol 1995, 5:497-500.

20. Linhares MI, Eizuru Y, Tateno S, Minamishima Y: Seroprevalence of human herpesvirus 6 infection in Brazilian and Japanese populations in the north-east of Brazil. Microbiol Immunol 1991, 35: 1023-7.

21. Zerr DM, Meier AS, Selke SS, Frenkel LM, Huang ML, Wald A, Rhoads MP, Nguy L, Borenemann R, Morrow RA, Corey L: A populationbased study of primary human herpesvirus 6 infection. $N$ Engl J Med 2005, 352(8):753-5.
Publish with Bio Med Central and every scientist can read your work free of charge

"BioMed Central will be the most significant development for disseminating the results of biomedical research in our lifetime. "

Sir Paul Nurse, Cancer Research UK

Your research papers will be:

- available free of charge to the entire biomedical community

- peer reviewed and published immediately upon acceptance

- cited in PubMed and archived on PubMed Central

- yours - you keep the copyright
BioMedcentral 